International Journal of Pure and Applied Mathematics

Volume 110 No. 2 2016, 265-273

ISSN: 1311-8080 (printed version); ISSN: 1314-3395 (on-line version)

url: http://www.ijpam.eu

doi: 10.12732/ijpam.v110i2.3

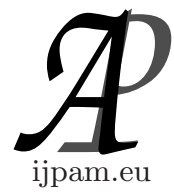

\title{
ANALYTICAL SOLUTION OF BAGLEY TORVIK EQUATION BY GENERALIZE DIFFERENTIAL TRANSFORM
}

\author{
Manish Kumar Bansal ${ }^{1 \S}$, Rashmi Jain ${ }^{2}$ \\ ${ }^{1,2}$ Department of Mathematics \\ Malaviya National Institute of Technology \\ Jaipur, 302017, INDIA
}

\begin{abstract}
In the present paper, we use generalized differential transform method (GDTM) to derive solution of Bagley Torvik equation. The fractional derivative are described in the Caputo sense. As an example we have found the exact solution of two such Bagley-Torvik equations which demonstrates the effectiveness and efficiency of the proposed method.
\end{abstract}

AMS Subject Classification: 26A33, 35A22, 65L05, 34A08

Key Words: Caputo fractional derivative, generalized differential transform method, BagleyTorvik equation, fractional differential equation

\section{Introduction}

The Bagley-Torvik equation is originally formulated to study the behavior of real material by use of fractional calculus $[2,20]$. It plays important role in many engineering and applied science problems. In particular, the equation with $1 / 2$ order derivative or $3 / 2$-order derivative can model the frequency-dependent damping materials quite satisfactorily. It can also describe motion of real physical systems, the modeling of the motion of a rigid plate immersed in a Newtonian fluid and a gas in a fluid, respectively $[14,17]$. Fractional dynamic systems have found many applications in various problems such as viscoelasticity, heat

Received: $\quad$ March 16, 2016

Revised: $\quad$ September 8, 2016

Published: November 4, 2016

$\S_{\text {Correspondence author }}$ (c) 2016 Academic Publications, Ltd. url: www.acadpubl.eu 
conduction, electrode-electrolyte polarization, electromagnetic waves, diffusion wave, control theory, and signal processing $[1,2,4,12,14,17,18,19,20,21]$.

The generic form of Bagley-Torvik equation [14, p. 229] can be written as

$$
A \frac{d^{2} y(t)}{d t^{2}}+B \frac{d^{\frac{3}{2}} y(t)}{d t^{\frac{3}{2}}}+C y(t)=f(t), \quad t>0
$$

Subject to initial conditions

$$
y(0)=0 \quad \text { and } \quad y^{\prime}(0)=0 .
$$

Here $y(t)$ is the solution of the equation, $A \neq 0$, $\mathrm{B}$, and $\mathrm{C}$ are constant coefficient's and $f(t)$ is a given function from $I$ into $R, I$ is the interval $[0, T]$. The analytic results on existence and uniqueness of solutions to fractional differential equations have been investigated by many authors $[14,16]$

The general response expression (1) contains parameters that can be varied to obtain various responses. In the case of $\mathrm{A}=\mathrm{M}$, the mass of thin rigid plate, $\mathrm{C}=\mathrm{K}$, the stiffness of the spring, $\mathrm{B}=2 S \sqrt{\mu \rho}$, where $\mathrm{S}$ is area of plate immersed in Newtonian fluid, $\mu$ is viscosity and $\rho$ is the fluid density, then (1) represents the motion of a large thin plate in a Newtonian fluid [14]. Similarly, linearly damped fractional oscillator with the damping term has a fractional derivative of order $\nu=1.5$ and it can be represented by Bagley-Torvik equation[13, 14]. The problem to develop the numerical solution of BagleyTorvik fractional differential equation has been studied by many authors. In this regard an approximate analytical solution of the equation was derived using Adomian decomposition method [6, 11], He's variational iteration method [10], Taylor collocation method [7], Laplace Transform method [14]. Diethelm transformed the equation into first-order coupled fractional differential equation and solved the problem with Adams predictor and corrector approach [5]. Podlubny used successive approximation method to solve the equation and recently applied the Matrix Approach to Discretization of Fractional Derivatives for the same problem $[14,15]$.

Recently several numerical methods have been proposed for solution of Bagley-Torvik equation [14]. In the present paper we obtain analytic solution of Bagley Torvik equation using generalized differential transform method [8]. The Differential Transform method was proposed by Zhou [22] to solve linear and nonlinear initial value problems in electric circuit analysis. This method constructs an analytical solution in the form of a series. It is different from the traditional higher order Taylor series method, which requires symbolic computation of the necessary derivatives of the data functions and takes long time 
in computation whereas the differential transform is an iterative procedure for obtaining analytic Taylor series solution. The method is further developed by Erturk, Momani and Odibat in their papers [8] for solving ordinary differential equations of fractional order.

\section{Preliminaries}

\subsection{Definition}

The Caputo fractional derivative use in our present study is defined and represented in the following manner [3]

$$
D_{x}^{\alpha} f(x)=\frac{1}{\Gamma(m-\alpha)} \int_{x_{0}}^{x} \frac{f^{(m)}(\xi)}{(x-\xi)^{\alpha-m+1}} d \xi, \quad(m-1<\alpha \leq m), m \in N
$$

The Generalized Differential Transform: Consider the generalized differential transform [8, p. 1647] of the $K^{\text {th }}$ derivative of function $\mathrm{f}(\mathrm{x})$ in one variable as follows:

$$
F_{\alpha}(k)=\frac{1}{\Gamma(\alpha k+1)}\left[\left(D_{x_{0}}^{\alpha}\right)^{k} f(x)\right]_{x=x_{0}}
$$

where $0<\alpha \leq 1,\left(D_{x_{0}}^{\alpha}\right)^{k}=D_{x_{0}}^{\alpha} \cdot D_{x_{0}}^{\alpha} \ldots D_{x_{0}}^{\alpha}(k-$ times $)$ and $F_{\alpha}(k)$ is the transformed function.

The Inverse Generalized Differential Transform of $F_{\alpha}(k)$ is defined in the following manner [8, p. 1647]

$$
f(x)=\sum_{k=0}^{\infty} F_{\alpha}(k)\left(x-x_{0}\right)^{\alpha k} .
$$

Some basic properties of the generalized differential transform given by equation (4) will also be required in the sequel:

Let $F_{\alpha}(k), G_{\alpha}(k)$ and $H_{\alpha}(k)$ be the generalized differential transforms of the functions $\mathrm{f}(\mathrm{x}), \mathrm{g}(\mathrm{x})$ and $\mathrm{h}(\mathrm{x})$ respectively, then:

(a) If $\mathrm{f}(\mathrm{x})=\mathrm{g}(\mathrm{x}) \pm \mathrm{h}(\mathrm{x})$, then $F_{\alpha}(k)=G_{\alpha}(k) \pm H_{\alpha}(k)$.

(b) If $\mathrm{f}(\mathrm{x})=\mathrm{ag}(\mathrm{x})$, then $F_{\alpha}(k)=a G_{\alpha}(k)$, where a is a constant.

(c) If $\mathrm{f}(\mathrm{x})=\mathrm{g}(\mathrm{x}) \mathrm{h}(\mathrm{x})$, then $F_{\alpha}(k)=\sum_{l=0}^{k} G_{\alpha}(l) H_{\alpha}(k-l)$. 
(d) If $\mathrm{f}(\mathrm{x})=D_{x_{0}}^{\alpha} g(x)$, then $F_{\alpha}(k)=\frac{\Gamma(\alpha(k+1)+1)}{\Gamma(\alpha k+1)} G_{\alpha}(k+1)$.

(e) If $\mathrm{f}(\mathrm{x})=\left(x-x_{0}\right)^{\gamma}, \gamma=n \alpha, n \in Z$, then $F_{\alpha}(k)=\delta(k-\gamma / \alpha)$, where

$$
\delta(k)=\left\{\begin{array}{cl}
1, & \text { if } \quad k=0 \\
0, & \text { otherwise }
\end{array}\right.
$$

(f) If $f(x)=D_{x_{0}}^{\beta} g(x), m-1<\beta \leq m$ and the function $\mathrm{g}(\mathrm{x})$ satisfies the condition in Theorem 2, then

$$
F_{\alpha}(k)=\frac{\Gamma(\alpha k+\beta+1)}{\Gamma(\alpha k+1)} G_{\alpha}(k+\beta / \alpha) .
$$

\subsection{Known Basic Theorems}

The following theorems will be required to obtain our main findings:

Theorem 1. (Generalized Taylor Formula) Suppose that $\left(D_{a}^{\alpha}\right)^{k} f(x) \in$ $C[a, b]$ for $k=0,1,2, \ldots n+1$, where $0<\alpha \leq 1$, then we have

$$
f(x)=\sum_{i=o}^{n} \frac{(x-a)^{i \alpha}}{\Gamma(i \alpha+1)}\left(\left(D_{a}^{\alpha}\right)^{i} f\right)(a)+\frac{\left(\left(D_{a}^{\alpha}\right)^{n+1} f\right)(\xi)}{\Gamma((n+1) \alpha+1)} \cdot(x-a)^{(n+1) \alpha},
$$

with $a \leq \xi \leq x, \forall \quad x \in[a, b]$. The above theorem was recently obtained by Erturk et al.[8].

Theorem 2. Suppose that $f(x)=\left(x-x_{0}\right)^{\lambda} g(x)$, where $\lambda>0$ and $g(x)$ has the generalized power series expansion $g(x)=\sum_{n=0}^{\infty} a_{n}\left(x-x_{0}\right)^{n \alpha}$ with radius of convergence $R>0,0<\alpha \leq 1$. Then

$$
D_{a}^{\gamma} D_{a}^{\beta} f(x)=D_{a}^{\gamma+\beta} f(x),
$$

for all $\left(x-x_{0}\right) \in(0, R)$, the coefficients $a_{n}=0$ for $n$ gives by $n \alpha-\lambda+\beta=0$ and either

$$
\lambda>\mu, \quad \mu=\max (\beta+[\gamma],[\beta+\gamma]),
$$

or

$$
\lambda \leq \mu, \quad a_{k}=0 \text { for } k=0,1,2, \ldots,\left[\frac{\mu-\lambda}{\alpha}\right],
$$

where [.] denotes the greatest integer less than or equal to $x$. The above theorem was recently obtained by Garg et al. [9]. 


\section{Application}

In this section, we shall apply GDTM for solving Bagley Torvik equation.

Consider the Bagley Torvik equation:

$$
A D^{2} y(t)+B D^{\frac{3}{2}} y(t)+C y(t)=f(t) \quad \text { where } t>0 .
$$

Subject to initial conditions

$$
y(0)=0 \quad \text { and } \quad y^{\prime}(0)=0,
$$

where $A \neq 0, \mathrm{~B}$ and $\mathrm{C}$ are constant and $\left(D_{a}^{\alpha}\right)^{k} f(x) \in C[a, b]$ for $k=0,1,2, \ldots, n+$ $1,0<\alpha \leq 1$ given in Theorem 1 .

Applying the Generalized Differential Transform on both sides of equation (8),we easily arrive at the following result after a little simplification

$$
A \frac{\Gamma(\alpha k+3)}{\Gamma(\alpha k+1)} Y_{\alpha}\left(k+\frac{2}{\alpha}\right)+B \frac{\Gamma\left(\alpha k+\frac{3}{2}+1\right)}{\Gamma(\alpha k+1)} Y_{\alpha}\left(k+\frac{3}{2 \alpha}\right)+C Y_{\alpha}(k)=F_{\alpha}(k),
$$

or

$$
Y_{\alpha}\left(k+\frac{2}{\alpha}\right)=\frac{F_{\alpha}(k)-C Y_{\alpha}(k)-B \frac{\Gamma\left(\alpha k+\frac{5}{2}\right)}{\Gamma(\alpha k+1)} Y_{\alpha}\left(k+\frac{3}{2 \alpha}\right)}{A(\alpha k+2)(\alpha k+1)} .
$$

Here $Y_{\alpha}(k)$ be the Generalized Differential Transform Function of $y(t)$.

Using the initial condition given by (9), the operator represented by eq. (4) takes the form

$$
\left[D_{t_{0}}^{\alpha k} y(t)\right]_{t=0}=0 \quad \text { for } k=0,1,2,3, \ldots .
$$

Applying the inverse generalized differential transform to equation (11), we obtain the desire solution with the help of eq.(12) after a little simplification.

\section{Examples}

In order to illustrate the effectiveness of the method proposed, two numerical examples are given in this section.

Example 1. Now we consider the following special case of Bagley-Torvik equation given by (1) as investigated earlier $[10,16]$

$$
D^{2} y(t)+D^{\frac{3}{2}} y(t)+y(t)=2+4 \sqrt{\frac{t}{\pi}}+t^{2} .
$$


Subject to initial conditions

$$
y(0)=0 \quad \text { and } \quad y^{\prime}(0)=0 .
$$

Applying generalized differential transform (4) with $t_{0}=0$ and settig $\alpha=1 / 2$ in equation (11) with condition (12), to both sides of Bagley -Torvik equation (13) and making use of properties of generalized differential transform given by (4), equation (13) takes the following form

$$
Y_{\frac{1}{2}}(k+4)=\frac{2 \delta(k)+\frac{4}{\bar{\pi}} \delta(k-1)+\delta(k-4)-Y_{\frac{1}{2}}(k)-\frac{\Gamma\left(\frac{k}{2}+\frac{5}{2}\right)}{\Gamma\left(\frac{k}{2}+1\right)} Y_{\frac{1}{2}}(k+3)}{\left(\frac{k}{2}+2\right)\left(\frac{k}{2}+1\right)},
$$

where

$$
Y_{\frac{1}{2}}(0)=0, Y_{\frac{1}{2}}(1)=0, Y_{\frac{1}{2}}(2)=0, Y_{\frac{1}{2}}(3)=0 .
$$

Using the recurrence relation (15) and the transformed initial condition (16), for $\mathrm{k}=1,2, \ldots$. we obtain after a little simplification the following result

$$
Y_{\frac{1}{2}}(k)= \begin{cases}1, & \text { if } \quad k=4, \\ 0, & \text { otherwise }(k \neq 4) .\end{cases}
$$

Again if in the inverse transform given by equation (5), we take $\alpha=\frac{1}{2}, t_{0}=0$ and $\mathrm{f}(\mathrm{x})$ replaced by $\mathrm{y}(\mathrm{t})$ then it takes the following form

$$
y(t)=\sum_{k=0}^{\infty} Y_{\frac{1}{2}}(k) t^{\frac{k}{2}}
$$

Using the values of $Y_{\frac{1}{2}}(k)$ From equation (17) in equation (18), a solution of Bagley-Torvik equation (13) is obtained as

$$
y(t)=t^{2} .
$$

it has been verified though we have obtained the solution $y(t)=t^{2}$ for $\alpha=\frac{1}{2}$, also we can obtain same solution for all values of $\alpha$ such that $0<\alpha \leq 1$.

Example 2. Now we consider the following special case of Bagley-Torvik equation given by (1) as investigated earlier $[6,7]$

$$
D^{2} y(t)+D^{\frac{3}{2}} y(t)+y(t)=t+1
$$

with initial condition

$$
y(0)=1 \quad \text { and } \quad y^{\prime}(0)=1 .
$$


Applying generalized differential transform (4) with $t_{0}=0$ and settig $\alpha=1 / 2$ in equation (11), to both sides of Bagley -Torvik equation (20) and making use of properties of generalized differential transform given by (4), equation (20) takes the following form

$$
Y_{\frac{1}{2}}(k+4)=\frac{\delta(k)+\delta(k-2)-Y_{\frac{1}{2}}(k)-\frac{\Gamma\left(\frac{k}{2}+\frac{5}{2}\right)}{\Gamma\left(\frac{k}{2}+1\right)} Y_{\frac{1}{2}}(k+3)}{\left(\frac{k}{2}+2\right)\left(\frac{k}{2}+1\right)},
$$

where

$$
Y_{\frac{1}{2}}(0)=1, Y_{\frac{1}{2}}(1)=0, Y_{\frac{1}{2}}(2)=1, Y_{\frac{1}{2}}(3)=0 .
$$

Using the recurrence relation (22) and the transformed initial condition (23), for $k=1,2, \ldots$, we obtain after a little simplification the following result

$$
Y_{\frac{1}{2}}(k)= \begin{cases}1, & \text { if } \quad k=0,2 \\ 0, & \text { otherwise }\end{cases}
$$

Again if in the inverse transform given by equation (5), we take $\alpha=\frac{1}{2}, t_{0}=0$ and $f(x)$ replaced by $y(t)$ then it takes the following form

$$
y(x)=\sum_{k=0}^{\infty} Y_{\frac{1}{2}}(k) x^{\frac{k}{2}} .
$$

Using the values of $Y_{\frac{1}{2}}(k)$ From equation (24) in equation (25), a solution of Bagley-Torvik equation (20) is obtained as

$$
y(t)=1+t
$$

It has been verified though we have obtained the solution $\mathrm{y}(\mathrm{t})=\mathrm{t}+1$ for $\alpha=\frac{1}{2}$, also we can obtain same solution for all values of $\alpha$ such that $0<\alpha \leq 1$.

\section{Conclusions}

Analytical exact solutions of two important special cases of Bagley Torvik equation, with fractional derivatives in Caputo sense, are obtained using generalized differential transform method. The present our study exhibits the potential of differential transform method in solving fractional differential equation in closed form. 


\section{Acknowledgment}

The authors are thankful to Prof. Mridula Garg for her valuable suggestions.

\section{References}

[1] Agarwal, R. P., Belmekki, M. and Benchohra, M. (2009). A survey on semi linear differential equations and inclusions involving Riemann-Liouville fractional derivative.Advances in Difference Equations, 2009(3), 1-47.

[2] Bagley, R. L. and Torvik, P. J.(1983). A different approach to the analysis of viscoelastically damped structures. AIAA Journal, 21(5), 741-748.

[3] Caputo, M.(1967). Linear models of dissipation whose Q is almost frequency independentII. Geophysical Journal International, 13(5), 529-539.

[4] Das, S.(2010). Solution of extraordinary differential equations with physical reasoning by obtaining modal reaction series. Modelling and Simulation in Engineering, 2010, 1-19.

[5] Diethelm, K. and Ford, N. J.(2002). Numerical solution of the Bagley-Torvik equation.BIT Numerical Mathematics, 42(3), 490-507.

[6] El-Sayed, A. M. A., El-Kalla, I. L. and Ziada, E. A. A.(2010). Analytical and numerical solutions of multiterm nonlinear fractional orders differential equations, Applied Numerical Mathematics, 60(8), 788-797.

[7] Enesiz, Y. C, Keskin, Y. and Kurnaz, A. (2010). The solution of the Bagley-Torvik equation with the generalized Taylor collocation method. Journal of the Franklin Institute Engineering and Applied Mathematics, 347(2), 452-466.

[8] Erturk, V.S., Momani, S. and Odibat, Z.(2008). Application of generalized differential transform method to multi-order fractional differential equations. Communications in Nonlinear Science and Numerical Simulation, 13(8), 1642-1654.

[9] Garg, M. and Manohar, P.(2015). Three-dimensional generalized differential transform method for space-time fractional diffusion equation, Palestine Journal of Mathematics, 4(1), 127-135.

[10] Ghorbani, A. and Alavi, A. (2008). Application of He's variational iteration method to solve semidifferential equations of nth order. Mathematical Problems in Engineering, 2008, 1-9.

[11] Hu, Y., Luo, Y. and Lu, Z.(2008). Analytical solution of the linear fractional differential equation by Adomian decomposition method. Journal of Computational and Applied Mathematics, 215(1), 220-229.

[12] Manabe, S.(2002). A suggestion of fractional-order controller for flexible spacecraft attitude control. Nonlinear Dynamics, 29(14), 251-268.

[13] Naber, M. (2010). Linear fractionally damped oscillator. International Journal of Differential Equations, 2010, 1-12.

[14] Podlubny, I.(1999).Fractional Differential Equations.California, USA: Academic Press.

[15] Podlubny, I., Skovranek, T. and Vinagre Jara, B. M.(2009). Matrix approach to discretization of fractional derivatives and to solution of fractional differential equations and their systems.Proceedings of the IEEE Conference on Emerging Technologies and Factory Automation, 1-6. 
[16] Rawashdeh, E.A.(2006). Numerical solution of semidifferential equations by collocation method.Applied Mathematics and Computation, 174(2), 869-876.

[17] Ray, S. S. and Bera, R. K. (2005). Analytical solution of the Bagley Torvik equation by Adomian decomposition method.Applied Mathematics and Computation, 168(1), 398410.

[18] Stevanovic Hedrih, K.(2006). The transversal creeping vibrations of a fractional derivative order constitutive relation of nonhomogeneous beam. Mathematical Problems in Engineering, 2006, 1-18.

[19] Tian, Y. and Chen, A. (2009). The existence of positive solution to three-point singular boundary value problem of fractional differential equation.Abstract and Applied Analysis, 2009, 1-18.

[20] Torvik, P. J. and Bagley, R. L. (1984). On the appearance of the fractional derivative in the behavior of real materials. Journal of Applied Mechanics, 51(2), 294-298.

[21] Zahoor, R. M. A. and Qureshi, I. M. (2009). A modified least mean square algorithm using fractional derivative and its application to system identification.European Journal of Scientific Research, 35(1), 14-21.

[22] Zhou, JK.(1986).Differential transformation and its applications for electrical circuits. Wuhan, China: Huazhong University Press. 
